\title{
The Application of Automated Microanalysis on SEM to the Characterization of Steel Samples
}

\author{
J. Goulden ${ }^{1}$, H. Jiang ${ }^{1}$, K. Thomsen ${ }^{2}$, K. Mehnert ${ }^{2}$, \\ 1. Oxford Instruments NanoAnalysis, Halifax Road, High Wycombe, HP12 3SE, UK \\ 2. ST Development ApS, Samsøgade 83, 8000 Aarhus, Denmark
}

The relationship between the mechanical and physical properties of metallic materials and microstructure is well documented; for example through the Hall-Petch relationship, where strength is inversely dependent on the square root of the grain diameter [1] and [2]. In this paper different steel types are analyzed to illustrate the applications and benefits of microanalysis in characterizing steels.

A fundamental parameter of steels is grain size, and many mechanical properties are improved by refining grain size. Accurate grain size measurement is therefore fundamentally important. This requires the accurate identification of all the grain boundaries and traditionally is measured using light optical microscopy (LOM). This typically involves a chemical etching of the steel surface to highlight the grain boundaries. However, in some cases chemical etching is complicated by a complex microsctructure where it is not possible to clearly reveal all the grain boundaries, and as a result accurate grain size cannot be determined.

An example of such a microstructure is shown in Figure 1, this is a hot rolled steel rod with a complex pearlitic microstructure; this fine lamellar structure, makes it difficult to reveal and identify all the grain boundaries by etching. In these cases EBSD is an alternative, as it detects grain boundaries by measuring crystallographic orientations, overcoming the limitations of image analysis and enabling an accurate determination of grain size. In addition, EBSD has the unique ability to identify special boundaries, or coincident site lattice boundaries which is essential for accurate grain size measurement in materials with a tendency to form twins (such as austenite).

In addition to being more accurate than LOM, the automation available in a modern EBSD system enables a significant amount of the sample surface to be quickly and readily analyzed, so that valid statistics can be obtained. Figure 2 shows a montaged grain map of the hot rolled steel rod; a quarter of the sample surface has been analyzed, from the centre to the edge, enabling a representative measure of grain size to be determined. This steel is used in the manufacture of bridge cables, where engineering the grain size was found to be key in the performance of the final steel wire.

Another important characteristic of steels is phase fraction identification and quantification. The relative fractions of different phases will influence the behavior of the steel in different regimes. In some cases individual or secondary phases are readily identified using X-ray microanalysis, or a combination of Xray analysis and EBSD. However, distinguishing crystallographically similar phases in steel is a recognized challenge with EBSD, as for example with marten site and ferrite. Here a technique which applies a new pattern quality measure to aid in identifying these phases is demonstrated. Different phases give a different range of pattern quality values. In the case of martensite and ferrite a bimodal distribution in pattern quality results from the presence of the relatively lower strain ferrite matrix (with a higher quality measure) and higher strain martensite. The use of this method to automatically quantify phase fractions is demonstrated. 


\section{References:}

[1] F. J. Humphreys, Journal of Materials Science 36 (2001) 3833.

[2] A. P. Day and T. E. Quested, Journal of Microscopy 195 (1999) 186.

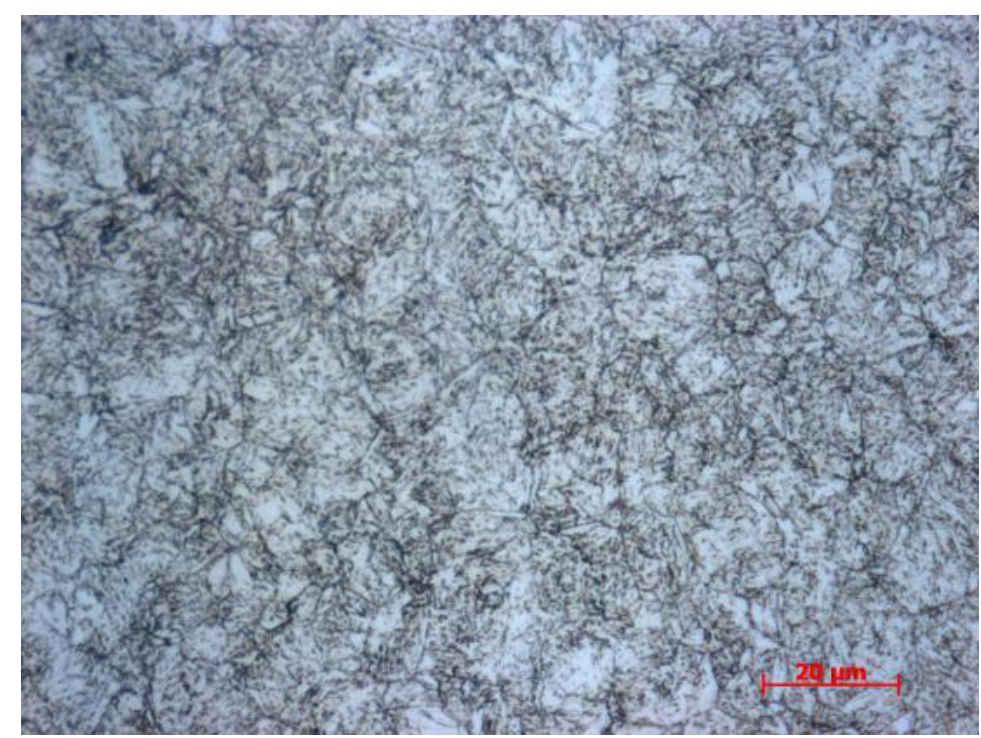

Figure 1 Optical micrograph showing difficult to distinguish pearlitic microstructure, making it difficult to distinguish the individual grains

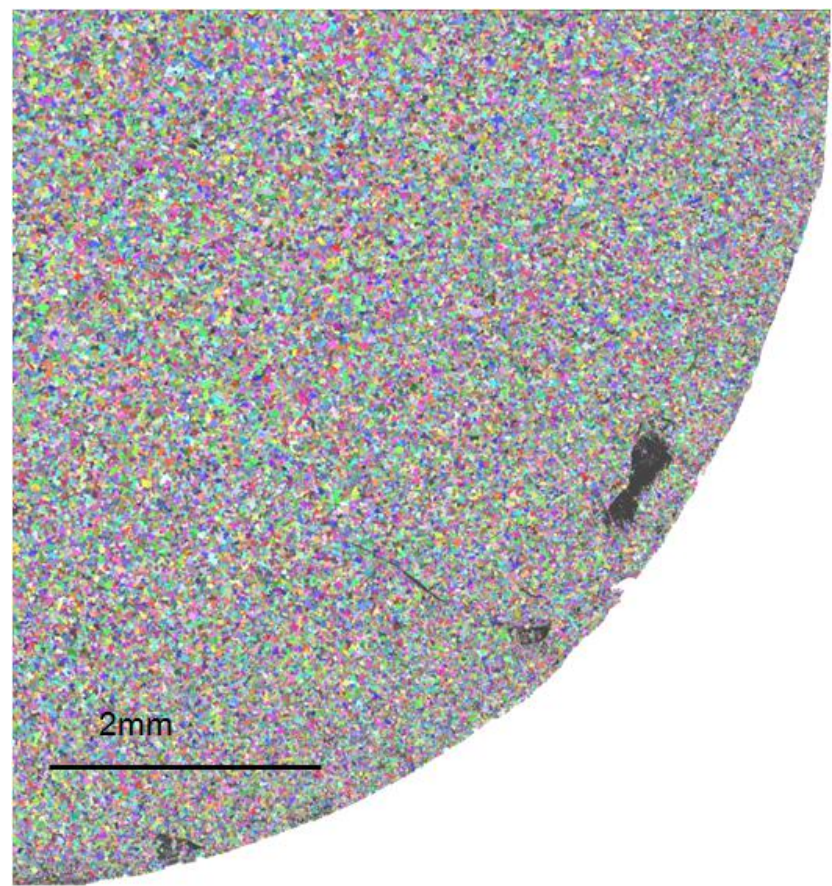

Figure 2 EBSD Grain Map, a montage of 132 individual fields, individual grains are identified based on orientation change. 